\title{
Two Cases of Lateral Lumbar Disc Herniation Successfully Treated with Intradiscal Condoliase Injection
}

\author{
Toru Funayama ${ }^{1)}$, Kentaro Mataki ${ }^{1)}$, Kohei Murakami ${ }^{2)}$, Yuki Mataki ${ }^{3)}$, Hiroshi Noguchi ${ }^{1)}$, Kousei Miura ${ }^{1)}$, Yosuke Shibao ${ }^{1)}$ \\ Fumihiko Eto ${ }^{1)}$, Mamoru Kono ${ }^{1)}$, Tetsuya Abe ${ }^{1)}$, Hiroshi Takahashi ${ }^{1)}$, Masao Koda ${ }^{1)}$ and Masashi Yamazaki ${ }^{1)}$ \\ 1) Department of Orthopaedic Surgery, Faculty of Medicine, University of Tsukuba, Tsukuba, Japan \\ 2) Department of Orthopaedic Surgery and Sports Medicine, Tsukuba University Hospital Mito Clinical Education and \\ Training Center, Mito Kyodo General Hospital, Mito, Japan \\ 3) Department of Orthopaedic Surgery, Ibaraki Prefectural University of Health Sciences, Ami, Japan
}

\begin{abstract}
Keywords:
lateral lumbar disc herniation, condoliase, intradiscal injection, conservative treatment

Spine Surg Relat Res 2021; 5(6): 437-441 dx.doi.org/10.22603/ssrr.2020-0127
\end{abstract}

Lateral lumbar disc herniation (LLDH) accounts for 7\%$12 \%$ of all lumbar disc herniations ${ }^{1-3)}$ and is more likely to occur in middle-aged to older adults ${ }^{4)}$. It is typically resistant to conservative therapy and often requires surgery due to compression on the dorsal root ganglion of the exiting nerve $\operatorname{root}^{2)}$.

An agent that induces chemical dissolution of the nucleus pulposus using condoliase (Hernicore ${ }^{\circledR}$, Kaken Pharmaceutical Co. $)^{5)}$ was recently approved as a novel intradiscal treatment for $\mathrm{LDH}^{6,7)}$. However, there is little information about its effectiveness in LLDH.

\section{Case 1}

A 77-year-old man with a history of unstable angina who was taking aspirin presented to our hospital complaining of one month of severe pain radiating to his left anterior thigh. The patient had a decreased left patellar tendon reflex. Manual muscle testing and sensations of the lower limbs were normal. The femoral nerve stretch test (FNST) was positive on the left. The Japanese Orthopaedic Association (JOA) score was $15 / 29$.

In the sagittal section of the T1 weighted MRI, the left L 3 nerve root was poorly visualized in the left L3/4 foramen due to a herniation (Fig. 1A). A horizontal section of T2 weighted MRI showed lateral disc herniation from inside to outside the left foramen at L3/4 (Fig. 1B). The degree of affected-disc degeneration was Grade IV, according to
Pfirrmann classification ${ }^{8)}$ (Fig. 1C). Standing radiography revealed that the L3/4 disc height was maintained (Fig. 1D). A CT after myelodiscography showed contrast media inflow to a left extraforaminal herniation at L3/4, but no intraforaminal or extraforaminal bony stenosis (Fig. 1E).

Due to the poor analgesic effect of oral drugs and selective nerve root blocks, we recommended surgery. However, the patient refused due to his heart condition and advanced age. Therefore, we injected $1.25 \mathrm{U}$ condoliase (Fig. 2A).

The pain improved within one week. One month after treatment, the patient did not need oral analgesics. An MRI three months after treatment showed the herniation had slightly decreased in size, and the left L3 nerve root was visualized (Fig. 2B, C). On standing radiography, the L3/4 disc height was slightly diminished (Fig. 2D). The JOA score had improved to $29 / 29$ points.

\section{Case 2}

A 69-year-old man had lingering left lower extremity paralysis after the surgical resection of a middle thoracic intradural extramedullary tumor. He began experiencing radiating severe pain on the right anterior thigh three weeks after surgery. He was taking rivaroxaban for postoperative deep vein thrombosis. Although myelopathy was persistent, FNST was positive on the right. The JOA score was 14/29.

In the sagittal section of the T1 weighted MRI, the right L3 nerve root was poorly visualized in the right L3/4 fora-

Corresponding author: Toru Funayama, funatoru3@md.tsukuba.ac.jp

Received: July 4, 2020, Accepted: August 12, 2020, Advance Publication: September 23, 2020

Copyright (C) 2021 The Japanese Society for Spine Surgery and Related Research 

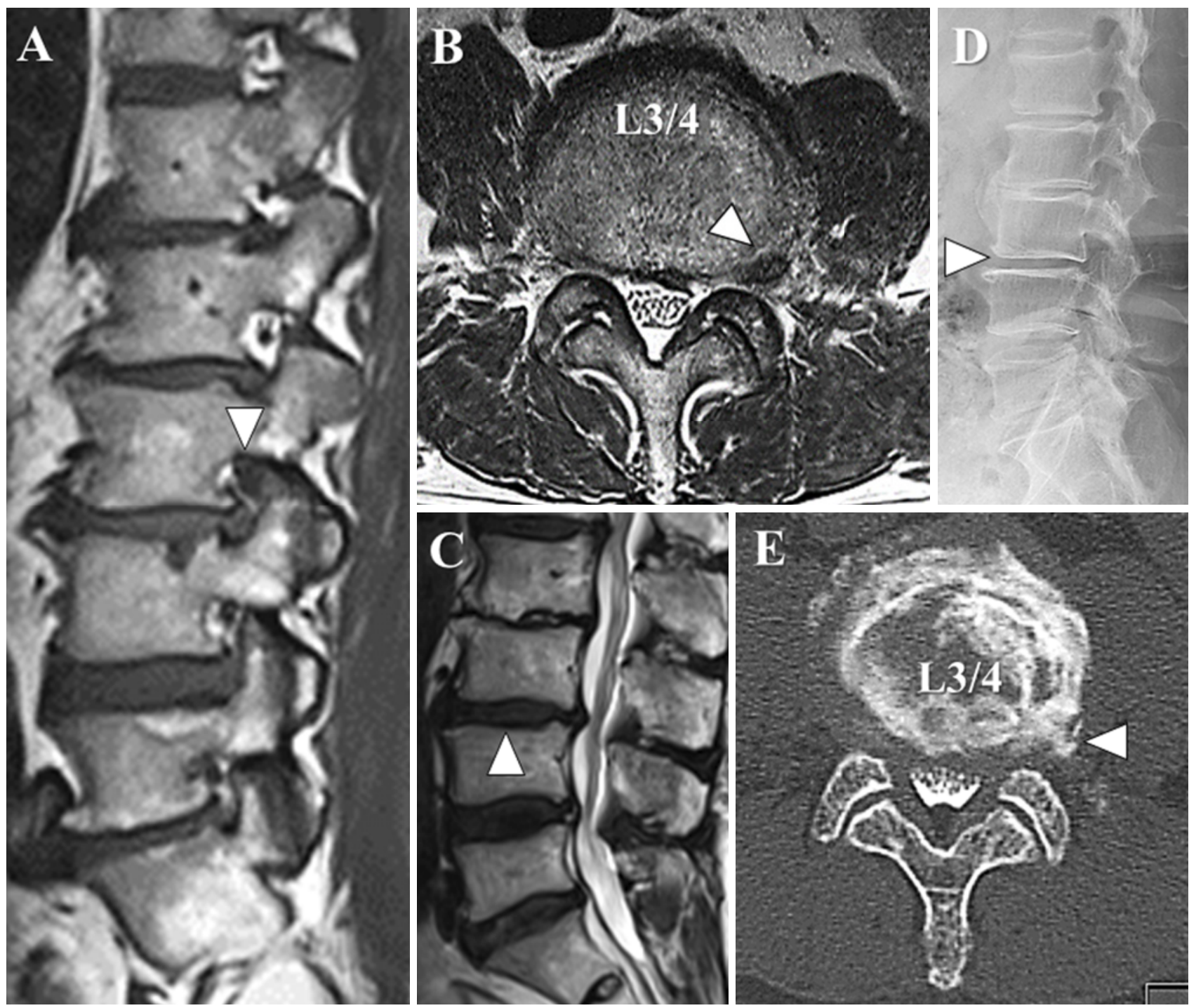

Figure 1. Before treatment of Case 1 .

In the sagittal section of the T1 weighted MRI, the left L3 nerve root was poorly visualized in the left L3/4 foramen due to a herniation (A, arrowhead). A horizontal section of T2 weighted MRI showed lateral disc herniation from inside to outside the left foramen at L3/4 (B, arrowhead). In the mid-sagittal section of a T2 weighted MRI, the degree of affected-disc degeneration was Grade IV, according to Pfirrmann classification ${ }^{8)}$ (C, arrowhead). Standing radiography revealed that the L3/4 disc height was maintained (D, arrowhead). A CT after myelodiscography showed inflow of contrast media into a left extraforaminal herniation at L3/4, but no intraforaminal or extraforaminal bony stenosis (E, arrowhead).

men due to a herniation (Fig. 3A). In the horizontal section of the T2 weighted MRI showed right extraforaminal disc herniation with high signal intensity at L3/4 (Fig. 3B). The degree of affected-disc degeneration was Grade III, according to Pfirrmann classification ${ }^{8}$ (Fig. 3C). Standing radiography showed that the L3/4 disc height was maintained (Fig. 3 D). A plain CT did not show osteophytes (Fig. 3E).

Due to the poor analgesic effect of oral drugs and selective nerve root blocks, the patient was unable to proceed with rehabilitation. Similar to case $1,1.25 \mathrm{U}$ condoliase was injected (Fig. 4A).

The pain had gradually decreased and had almost disappeared after 10 days. An MRI performed four months after treatment showed that the herniation had slightly decreased in size, and the right L3 nerve root could be visualized (Fig. 4B, C). On standing radiography, the L3/4 disc height was slightly diminished (Fig. 4D). The JOA score had improved to $21 / 29$ points.

Condoliase injected into an intervertebral disc specifically dissolves glycosaminoglycans, which constitute proteoglycans, the main component of the nucleus pulposus ${ }^{6}$. This restricts the proteoglycans' water-holding capacity, reducing pressure inside the intervertebral disc, lowering the pressure on the nerve root from the hernia, and improving lower extremity and low back pain ${ }^{6}$. A previous study observed significant analgesic effects, improved lumbar function, and improved quality of life compared to a placebo in cases of protrusion-type or subligamentous-type lumbar disc hernia- 

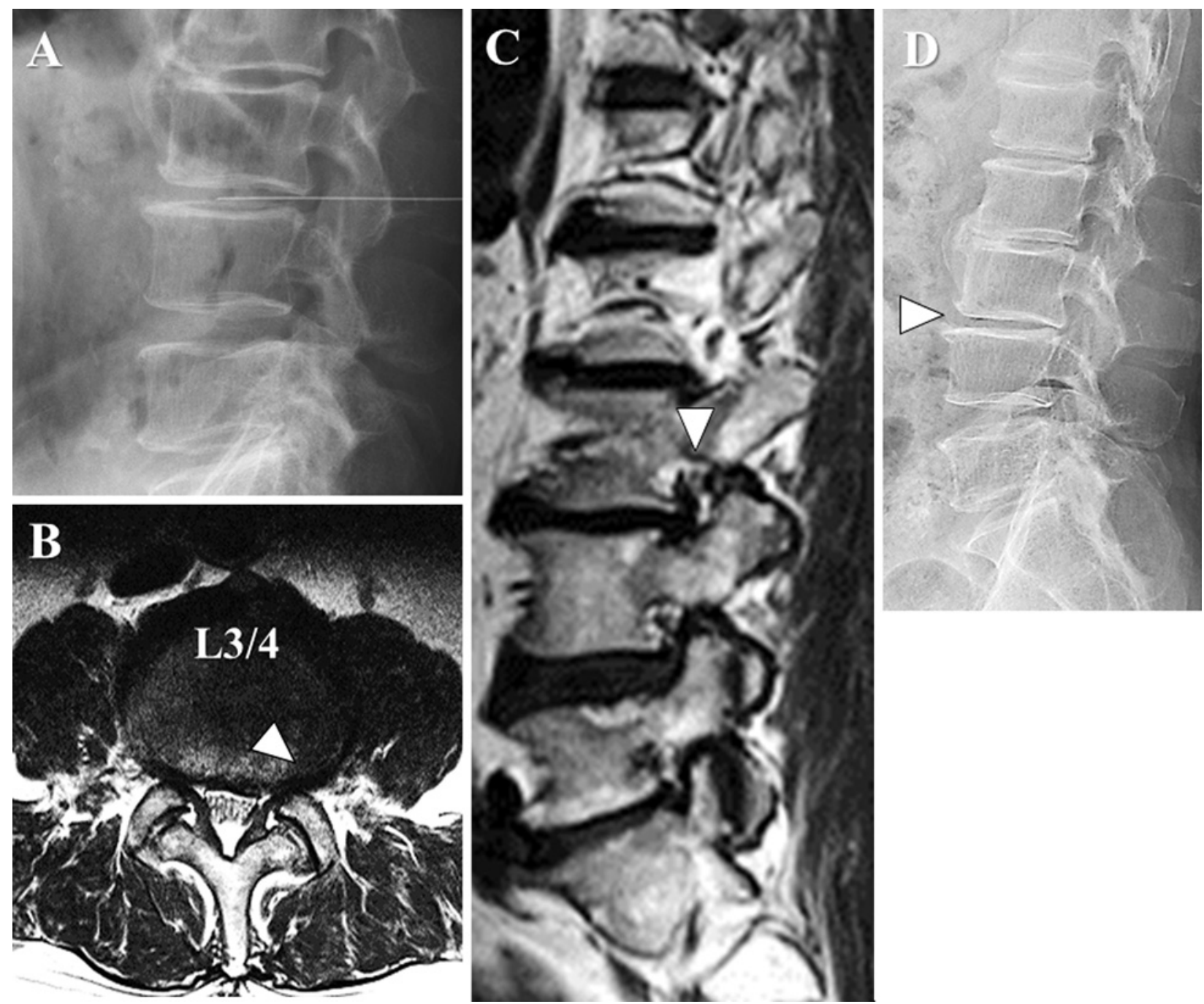

Figure 2. During and after treatment of Case 1.

In the prone position, a $12 \mathrm{~cm}$ spinal needle was inserted from the right-lateral side under fluoroscopy and advanced to the center of the $\mathrm{L} 3 / 4$ disc, where $1.25 \mathrm{U}$ condoliase dissolved in $1.2 \mathrm{~mL}$ of physiological saline was injected (A). A lumbar MRI three months after treatment showed that the left extraforaminal herniation at L3/4 had slightly decreased in size in the horizontal section of a T2 weighted image (B, arrowhead) and the left L3 nerve root in the L3/4 foramen was visualized in sagittal section of T1 weighted image (C, arrowhead). On standing radiography, the L3/4 disc height was slightly diminished (D, arrowhead).

tion inside the spinal canal $^{7}$. Another study concluded that condoliase injections seem to be most effective for transligamentous type and herniation with high signal change on the T2 weighted image ${ }^{9)}$. They also indicated that there was no significant difference in affected-disc degeneration between efficacious and inefficacious group ${ }^{9)}$.

This study is the first report on condoliase injections for LLDH. In both cases, conservative therapy with oral medications and selective nerve root block were not successful. However, intradiscal condoliase injections were effective, achieving an analgesic effect soon after treatment and allowing elderly patients to avoid surgery. In case 1, although the affected-disc degeneration had progressed, the disc height was maintained. Therefore, there seemed to be contained nucleus pulposus component in the herniated disc. In case 2, the disc herniation showed high signal change on the T2 weighted image, which is the better condition for condoliase injection therapy ${ }^{9}$.

Although more findings from large case series or comparative studies are needed, this report suggests that intradiscal condoliase injection could be a useful, novel conservative treatment option for LLDH.

Conflicts of Interest: The authors declare that there are no relevant conflicts of interest.

\section{Sources of Funding: None.}

Author Contributions: Toru Funayama wrote and prepared the manuscript, and all of the authors participated in the study design. All authors have read, reviewed, and approved the article.

Ethical Approval: Unnecessary for Clinical Correspon- 

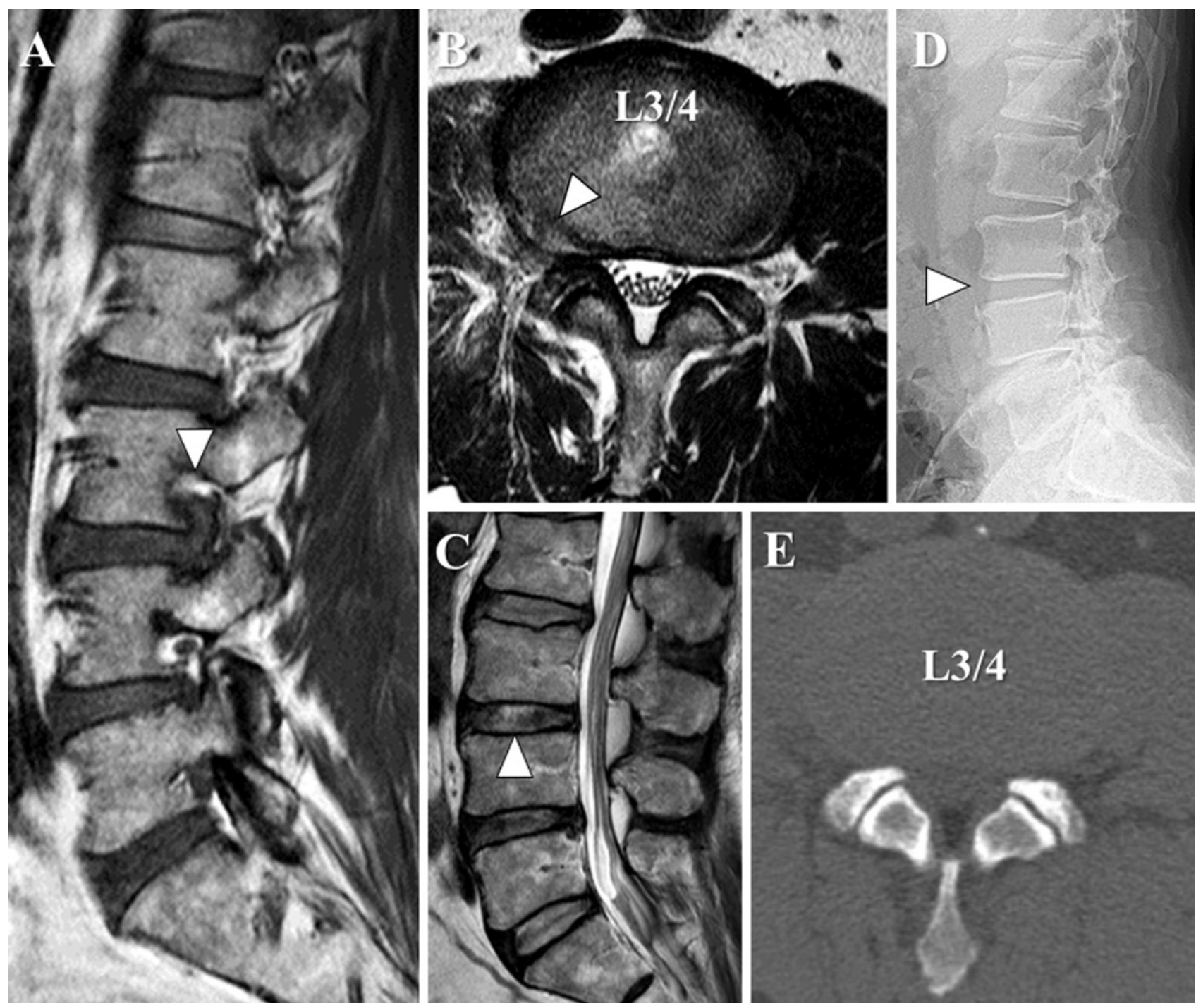

Figure 3. Before treatment of Case 2 .

In the sagittal section of a T1 weighted MRI, the right L3 nerve root was poorly visualized in the right L3/4 foramen due to a herniation (A, arrowhead). In the horizontal section of a T2 weighted MRI showed right extraforaminal disc herniation with high signal intensity at L3/4 (B, arrowhead). In the mid-sagittal section of T2 weighted MRI, the degree of affected-disc degeneration was Grade III, according to Pfirrmann classification ${ }^{8)}$ (C, arrowhead). Standing radiography showed that the L3/4 disc height was maintained (D, arrowhead). A plain CT did not show osteophytes at L3/4 (E).

dence.

Informed Consent: Informed consent was obtained by the patients in this study.

\section{References}

1. Marquard G, Bruder M, Theuss S, et al. Ultra-long-term outcome of surgically treated far-lateral, extraforaminal lumbar disc herniations: a single-center series. Eur Spine J. 2012;21(4):660-5.

2. Epstein NE. Foraminal and far lateral lumbar disc herniations: surgical alternatives and outcome measures. Spinal Cord. 2002;40 (10):491-500

3. Salame K, Lidar Z. Minimally invasive approach to far lateral lumbar disc herniation: technique and clinical results. Acta Neurochir. 2010;152(4):663-8.

4. Lee JH, Lee SH. Clinical and radiological characteristics of lum- bosacral lateral disc herniation in comparison with those of medial disc herniation. Medicine. 2016;95(7):e2733.

5. Matsuyama Y, Chiba K. Condoliase for treatment of lumbar disc herniation. Drugs Today. 2019;55(1):17-23.

6. Matsuyama Y, Chiba K, Iwata $\mathrm{H}$, et al. A multicenter, randomized, double-blind, dose-finding study of condoliase in patients with lumbar disc herniation. J Neurosurg Spine. 2018;28(5):499-511.

7. Chiba K, Matsuyama Y, Seo T, et al. Condoliase for the treatment of lumbar disc herniation: a randomized controlled trial. Spine. 2018;43(15):E869-76.

8. Pfirrmann C, Metzdorf A, Zanetti M, et al. Magnetic resonance classification of lumbar intervertebral disc degeneration. Spine. 2001;26(17):1873-8.

9. Banno T, Hasegawa T, Yamato Y, et al. Clinical outcome of condoliase injection treatment for lumbar disc herniation: Indications for condoliase therapy. J Orthop Sci. 2020. 

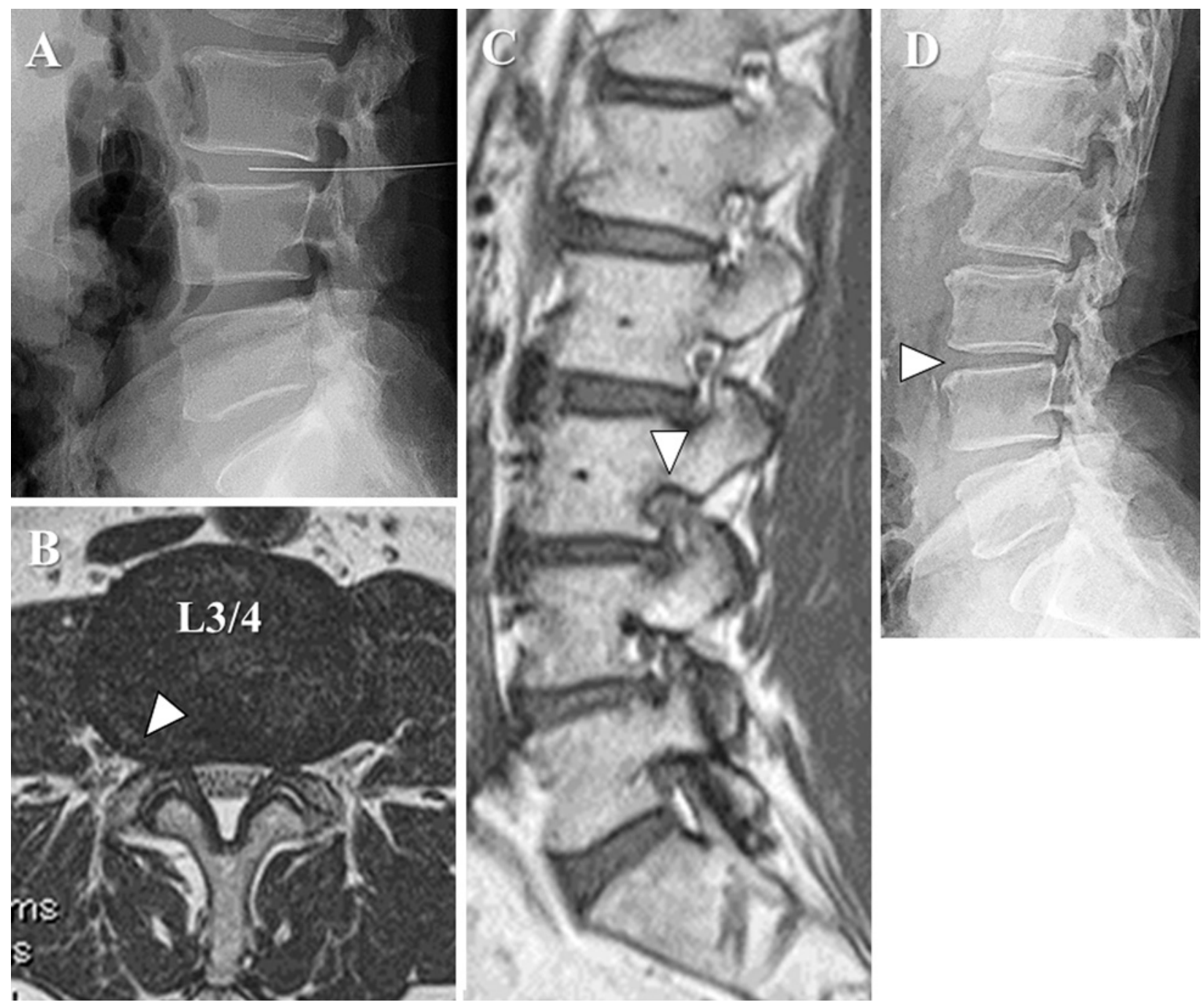

Figure 4. During and after treatment of Case 2.

Similar to case 1, $1.25 \mathrm{U}$ condoliase was injected into the intervertebral disc from the left-lateral side (A). A lumbar MRI performed 4 months after treatment showed that the right extraforaminal herniation at L3/4 had slightly decreased in size in the horizontal section of T2 weighted image (B) and the right L3 nerve root in the L3/4 foramen could be visualized in the sagittal section of T1 weighted image (C, arrowhead). On standing radiography, the L3/4 disc height was slightly diminished (D, arrowhead). 